\title{
Artigo/Article
}

\section{Associação entre dissinergia miocárdica e arritmia ventricular na forma indeterminada da doença de Chagas}

\author{
Association between left ventricular wall motion abnormalities and ventricular arrhythmia in \\ the indeterminate form of Chagas disease
}

Márcio Lins Barros $^{1,2}$, Antonio Luiz Ribeiro ${ }^{1,2}$, Maria do Carmo Nunes ${ }^{1,2}$ e Manoel Otávio da Costa Rocha ${ }^{1,2}$

\begin{abstract}
RESUMO
Introdução: Pacientes na forma indeterminada da doença de Chagas (FIDC) podem apresentar anormalidades contráteis segmentares, com evidências de pior prognóstico comparativamente a pacientes com estudo ecocardiográfico normal. O objetivo deste estudo foi de avaliar a associação entre a presença de anormalidade contrátil segmentar e a presença de distúrbios do ritmo cardíaco na FIDC. Métodos: Foram estudados 38 pacientes na FIDC, sendo 26 pacientes sem anormalidade contrátil e 12 com presença de distúrbio contrátil ao estudo ecocardiográfico. Todos os pacientes foram submetidos a um estudo ecocardiográfico completo, incluindo o Doppler tecidual, bem como à monitorização eletrocardiográfica de 24h (Holter). Resultados: Entre as diversas variáveis estudadas, foram encontradas diferenças significativas entre os dois grupos em relação à fração de ejeção $(p \leq 0,001)$, dimensão sistólica do ventrículo esquerdo $(p=0,029)$ e tempo de contração isovolumétrica $(\mathrm{p}<0,05)$ medidos pela ecocardiografia e Doppler tecidual, bem como extrassistolia ventricular isolada $(p=0,016)$ e em pares $(p=0,003)$ pela avaliação pelo Holter. Conclusões: Pacientes na FIDC que apresentam anormalidades contráteis quando avaliados pela ecocardiografia apresentam episódios mais frequentes de extrassistolia ventricular, traduzindo um maior dano morfofuncional e elétrico do coração, quando comparados a pacientes que apresentem estudo ecocardiográfico normal.
\end{abstract}

Palavras-chaves: Doença de Chagas. Ecocardiografia. Arritmia ventricular.

\begin{abstract}
Introduction: Chagas' disease can lead to severe and potentially lethal damage of cardiac function. Thus, the identification of cardiac abnormalities in patients presenting the indeterminate form (IF) can be important for risk stratification. This paper aimed to demonstrate whether IF chagasic patients who presented wall motion abnormalities showed functional and electric disturbances compared to patients with normal echocardiogram and Doppler studies. Methods: Thirty eight patients with the IF were studied, including 26 chagasic patients with normal electrocardiographic, radiological and echocardiographic exams and 12 chagasic patients showing segmental wall motion abnormalities in the echocardiographic exam. All the patients were submitted to complete echocardiogram and Doppler study, including tissue Doppler imaging (TDI) and Holter 24h monitoring. Results: Among the variables evaluated, significant differences among the two groups were verified in relation to: the ejection fraction ( $\mathrm{p} \leq 0.001)$; left ventricular systolic dimension $(\mathrm{p}=0.029)$; isovolumic contraction time, measured by TDI in the basal segments of the left ventricle $(\mathrm{p}<0.05)$; and the presence of isolated $(p=0.0005)$ and paired ventricular extrasystoles $(p=0.003)$, in the Holter monitoring. Conclusions: The indeterminate form of Chagas' disease can present cardiac wall motion abnormality, demonstrating functional and electric damage compared to chagasic patients with normal echocardiogram.
\end{abstract}

Keywords: Chagas disease. Echocardiography. Ventricular arrhythmia.

\footnotetext{
1. Hospital das Clínicas, Universidade Federal de Minas Gerais, Belo Horizonte, MG. 2. Programa de Pós-Graduação em Infectologia e Medicina Tropical, Faculdade de Medicina, Universidade Federal de Minas Gerais, Belo Horizonte, MG.

Endereço para correspondência: Dr. Márcio Vinícius Lins Barros. HC/UFMG. Rua Carangola 57/1201, Santo Antônio, 30330-240 Belo Horizonte, MG.

Tel: 5531 3292-2207

e-mail: marciovlbarros@uol.com.br

Recebido para publicação em 26/12/2009

Aceito em 26/11/2010
}

\section{INTRODUÇÃO}

A doença de Chagas representa uma das principais causas de morbimortalidade na América Latina, com grande impacto socioeconômico gerado pelo acometimento de expressivo contingente populacional, especialmente, na faixa mais produtiva da vida ${ }^{1}$. Aproximadamente, metade destes pacientes não apresenta sinais clínicos, eletrocardiográficos e radiológicos de envolvimento cardíaco, embora parte não desprezível desta população desenvolva cardiopatia ao longo dos anos ${ }^{2}$. Embora esses pacientes não demonstrem redução significativa da função sistólica global ventricular, estudos prévios têm demonstrado evidência de anormalidade contrátil segmentar em indivíduos na forma indeterminada da doença de Chagas (FIDC), com evidência de prognóstico desfavorável comparativamente a pacientes que não apresentem dissinergia ventricular ${ }^{3}$. O objetivo deste trabalho foi de estudar, comparativamente, através da ecocardiografia e da monitorização eletrocardiográfica de $24 \mathrm{~h}$ (Holter), o comportamento morfofuncional e elétrico cardíaco em pacientes na forma indeterminada da doença de Chagas que apresentem ou não distúrbios da contratilidade ventricular esquerda.

\section{MÉTODOS}

Foram selecionados 38 pacientes, em um estudo transversal e com coleta prospectiva de dados, sendo 18 do sexo feminino e 20 do masculino, selecionados em dois grupos: 0-chagásicos na FIDC e com ecocardiograma normal (26 pacientes) e 1-chagásicos na FIDC e com anormalidade contrátil ventricular esquerda (12 pacientes). Todos os pacientes foram selecionados no Centro de treinamento e Referência em Doenças Infecciosas e Parasitárias (CTR-DIP) da Universidade Federal de Minas Gerais (UFMG) após esclarecimento individual sobre os objetivos da investigação e consentimento escrito, pós informado. Para a realização do recrutamento, os pacientes foram inicialmente submetidos à anamnese e exame físico 
padronizados, eletrocardiograma, radiografia de tórax e exames laboratoriais (hemograma completo, dosagem bioquímica de creatinina, uréia, glicemia de jejum, potássio e sódio, dosagem de TSH e exames sorológicos para infecção pelo Trypanosoma cruzi por pelo menos três métodos diferentes), radiologia do esôfago e cólons. Foram considerados chagásicos com a forma indeterminada, aqueles pacientes que apresentaram positividade sorológica para Trypanosoma cruzi através de pelo menos duas entre técnicas diferentes (reação de imunofluorescência indireta, hemaglutinação indireta e ELISA), além de apresentar ECG, radiografia do tórax e radiologia do esôfago e cólons normais.

Foram considerados critérios de exclusão: hipertensão arterial sistêmica, definida como pressão arterial medida durante o exame físico $\geq 160 / 95$; história sugestiva de insuficiência coronariana; episódio prévio sugestivo de doença reumática; diabetes mellitus ou intolerância à glicose, conforme definido pelo National Diabetes Data Group; disfunção tireoidiana, manifesta por níveis anormais de hormônio estimulante da tireóide (TSH) e da tiroxina (T4) livre; insuficiência renal, definida por aumento dos níveis de creatinina e uréia; doença pulmonar obstrutiva crônica, a partir de dados clínicos, radiológicos e eletrocardiográficos sugestivos; distúrbios hidroeletrolíticos, a partir das dosagens de sódio e potássio; anemia significativa, definida como hemoglobina abaixo de $10 \mathrm{~g} / \mathrm{dl}$; alcoolismo, definido como consumo médio semanal de $420 \mathrm{~g}$ de etanol; gravidez; impossibilidade de realização do protocolo.

Os pacientes foram submetidos a ecodopplercardiograma com mapeamento de fluxo em cores, utilizando aparelho ATL HDI 5.000 (Bothell, Washington, USA), para obtenção dos diversos índices de função sistodiastólica enumerados a seguir: dimensões diastólica e sistólica finais do ventrículo esquerdo e diâmetro do átrio esquerdo; obtenção da fração de ejeção pelo método de Simpson, a partir de software fornecido pelo aparelho. Para análise da função diastólica, foi utilizado o Doppler pulsado do fluxo transmitral (ondas E, A, relação E/A e tempo de desaceleração- TD) e da via de saída do ventrículo esquerdo (tempo de relaxamento isovolumétrico- TRIV). O Doppler tissular foi obtido utilizando a janela apical nos cortes apical 4 câmaras, apical 2 câmaras e apical longo para avaliação das paredes septal, lateral, inferior, anterior e posterior, posicionando-se o volume amostra do cursor Doppler ao nível das porções basal, médio e apical das referidas paredes a fim de se determinar as velocidades de contração, medindo-se a velocidade de pico sistólica, além da estimativa do tempo de contração isovolumétrica regional ( $\mathrm{TCIVr}$ ), medindo-se o intervalo entre a onda $\mathrm{Q}$ do eletrocardiograma e o início da onda sistólica.

A monitorização eletrocardiográfica de $24 \mathrm{~h}$ foi realizada utilizando um sistema de gravação portátil de três canais (Dynamis, Cardios, São Paulo, Brazil). Os pacientes foram estimulados a desenvolverem suas atividades normais durante o período de gravação. As gravações foram analisadas no sistema Burdick/DMI Hospital Holter System (Spacelabs Burdick, Deerfield, WI, USA) por uma técnica semiautomática, com frequência de amostragem de $200 \mathrm{~Hz}$. Os traçados foram revistos por especialista experiente (ALPR), que realizou a codificação dos batimentos ectópicos e a exclusão de artefatos, gerando relatório consubstanciado. Foram avaliadas, especificamente neste estudo, as frequências cardíacas média, mínima e máxima, o número de extra-sístoles totais, número de pares e número de episódios de taquicardia ventricular não sustentada.
Os dados clínicos e gerados pelos procedimentos foram analisados utilizando o pacote estatístico SPSS versão 16 para ambiente Windows. Inicialmente, foi realizada a estatística descritiva das variáveis escolhidas, obtendo-se a distribuição de frequência absoluta e relativa das variáveis qualitativas e a média e desvio padrão ou mediana e intervalo interquartil das variáveis quantitativas contínuas, dependendo da distribuição, se normal ou não. A seguir, comparam-se as proporções, médias e medianas das variáveis independentes e dependentes dos grupos, através de testes estatísticos apropriados, como o qui-quadrado, para variáveis qualitativas, e os testes $t$ de Student e Mann-Whitney, para variáveis quantitativas, com distribuição normal ou não-normal, respectivamente. Em todos os testes, utilizou-se a probabilidade de significância $\mathrm{p}<0,05$ como necessário para rejeição da hipótese nula.

\section{Considerações éticas}

Este estudo foi aprovado pelo Comitê de Ética em Pesquisa da UFMG, sendo consideradas as recomendações da Organização Mundial de Saúde e da Declaração de Helsinque de 2000, no sentido de se salvaguardarem os direitos e o bem estar das pessoas estudadas.

\section{RESULTADOS}

Os resultados dos parâmetros gerais obtidos dos ecocardiogramas realizados nos dois grupos estão sumariados na Tabela 1. Dentre estas variáveis, observa-se diferença estatisticamente significativa relação à fração de ejeção $(\mathrm{p} \leq 0,001)$ e dimensão sistólica do ventrículo esquerdo $(\mathrm{p}=0,029)$. Dentre os pacientes analisados, com a ecocardiografia, $28,7 \%$ apresentaram alteração da contratilidade segmentar, com predominância pelo acometimento do ápex ventricular $(76,1 \%)$, sendo encontrado, nesta região, aneurisma de pequena dimensão, em três $(6,5 \%)$ pacientes. A análise pelo Doppler tecidual não demonstrou diferença significativa em relação às velocidades sistólica e diastólica, com diferença estatisticamente significativa, em relação ao TCIV avaliado nos segmentos basais, de todas as paredes, excetuando-se a parede lateral (Tabela 2).

TABELA 1 - Dados gerais e ecocardiográficos observados em 38 pacientes na forma indeterminada da doença de Chagas, sem (grupo 0) e com (grupo 1) dissinergia segmentar miocárdica.

\begin{tabular}{lccc}
\hline Variável* $^{*}$ & $\begin{array}{c}\text { Grupo } 0 \\
\mathrm{n}^{\circ}=26\end{array}$ & $\begin{array}{c}\text { Grupo } 1 \\
\mathrm{n}^{\circ}=12\end{array}$ & $\mathrm{P}$ \\
\hline Idade & $37,9 \pm 2,8$ & $39,7 \pm 8,9$ & 0,66 \\
\hline DDVE $(\mathrm{mm})$ & $49,2 \pm 3,0$ & $50,1 \pm 4,8$ & 0,48 \\
\hline DSVE $(\mathrm{mm})$ & $31,8 \pm 2,5$ & $34,5 \pm 4,9$ & 0,029 \\
\hline AE $(\mathrm{mm})$ & $33,4 \pm 1,8$ & $33,8 \pm 1,4$ & 0,53 \\
\hline FE $(\%)$ & $64,1 \pm 2,8$ & $59,1 \pm 3,9$ & $<0,001$ \\
\hline E/A & $1,5 \pm 0,3$ & $1,3 \pm 0,4$ & 0,49 \\
\hline E $(\mathrm{cm} / \mathrm{s})$ & $50,3 \pm 5,3$ & $49,4 \pm 6,2$ & 0,62 \\
\hline A $(\mathrm{cm} / \mathrm{s})$ & $34,0 \pm 7,7$ & $32,1 \pm 5,9$ & 0.34 \\
\hline Dt $(\mathrm{m} / \mathrm{s})$ & $181,1 \pm 18,9$ & $187,8 \pm 17,8$ & 0,38 \\
\hline TRIV $(\mathrm{m} / \mathrm{s})$ & $86,2 \pm 6,2$ & $87,4 \pm 7,1$ & 0,19 \\
\hline
\end{tabular}

*variáveis descritas por média \pm desvio padrão. DDVE: diâmetro diastólico do ventrículo esquerdo, DSVE: diâmetro sistólico do ventrículo esquerdo, $\mathrm{AE}$ : diâmetro do átrio esquerdo, FE: fração de ejeção, E/A: relação entre as ondas E e A, E: onda de enchimento rápido do fluxo transmitral, A: fluxo de contração atrial, Dt: tempo de desaceleração, TRIV: tempo de relaxamento isovolumétrico. 
TABELA 2 - Tempo de contração isovolumétrica pelo Doppler tecidual observados em 38 pacientes na forma indeterminada da doença de Chagas, sem (grupo 0) e com (grupo 1) dissinergia segmentar miocárdica.

\begin{tabular}{lccc}
\hline Parede do ventrículo esquerdo & $\begin{array}{c}\text { Grupo } 0 \\
\mathrm{n}^{\circ}=26\end{array}$ & $\begin{array}{c}\text { Grupo } 1 \\
\mathrm{n}^{\circ}=12\end{array}$ & $\mathrm{p}$ \\
\hline Septal & $92,7 \pm 10,0$ & $110,0 \pm 13,8$ & 0,001 \\
\hline Lateral & $88,5 \pm 12,5$ & $100,0 \pm 20,7$ & 0,069 \\
\hline Inferior & $90,1 \pm 9,0$ & $108,2 \pm 24,7$ & 0,006 \\
\hline Posterior & $91,2 \pm 10,9$ & $104,9 \pm 20,2$ & 0,023 \\
\hline Anterior & $89,3 \pm 10,0$ & $102,6 \pm 24$ & 0,023 \\
\hline
\end{tabular}

*variáveis descritas por média \pm desvio padrão.

A Tabela 3 demonstra dados relacionados com o Holter entre os dois grupos. Entre as variáveis estudadas, foi observado um maior número de extrassistolia ventricular isolada e pareada, denotando um maior distúrbio elétrico em pacientes chagásicos com dissinergia segmentar.

TABELA 3 - Monitorização ambulatorial de 24h (Holter) observados em 38 pacientes na forma indeterminada da doença de Chagas, sem (grupo 0 ) e com (grupo 1) dissinergia segmentar miocárdica.

\begin{tabular}{lccc}
\hline Variável $^{*}$ & $\begin{array}{c}\text { Grupo 0 } \\
\mathrm{n}^{\circ}=25\end{array}$ & $\begin{array}{c}\text { Grupo } 1 \\
\mathrm{n}^{\circ}=12\end{array}$ & $\mathrm{p}$ \\
\hline FC máxima (bpm) & $140(124-154)$ & $147(121-158)$ & 0,73 \\
\hline FC mínima (bpm) & $46(44-52)$ & $46(45-52)$ & 0,95 \\
\hline FC média (bpm) & $74(69-81)$ & $73(66-80)$ & 0,47 \\
\hline ES ventriculares (n⿳o em 24h) & $4(0-64)$ & $45(20-992)$ & 0,016 \\
\hline ES ventriculares (>10 por 24h: $\mathrm{n}^{\circ}(\%)$ & $9(36,0)$ & $11(92,0)$ & 0,002 \\
\hline ES ventriculares pareadas: $\mathrm{n}^{\circ}(\%)$ & $3(12,0)$ & $7(58,3)$ & 0,003 \\
\hline TVNS: $\mathrm{n}^{\circ}(\%)$ & $1(4,0)$ & $1(9,1)$ & 0,56 \\
\hline
\end{tabular}

*valores expressos pela mediana (interquartil) e, quando indicado, pelo número absoluto e percentagem. FC: frequência cardíaca, ES: extrassístoles, TVNS: taquicardia ventricular não sustentada.

\section{DISCUSSÃO}

A doença de Chagas é causada pelo protozoário Trypanosoma cruzi e representa uma das principais causas de morte em diversos países da América Latina ${ }^{1}$. O coração é o órgão mais frequente e severamente acometido, podendo determinar a morte por arritmia, insuficiência cardíaca ou por fenômenos tromboembólicos. Cerca de 50\% dos pacientes infectados encontram-se na forma crônica indeterminada e embora a característica principal destes pacientes seja a ausência de anormalidades clínicas, eletrocardiográficas e radiológicas significativas, tem-se observado alterações morfofuncionais cardíacas quando se utilizam métodos complementares mais sofisticados. Embora vários estudos longitudinais tenham demonstrado o bom prognóstico dos pacientes na forma indeterminada, cerca de 2 a $5 \%$ destes evoluem anualmente para uma das outras formas crônicas sendo que, dentro de cinco a 10 anos, $30 \%$ destes pacientes evoluem para cardiopatia crônica ${ }^{2}$. Além disso, tem-se relatado a presença de morte súbita em chagásicos como a primeira manifestação clínica da doençç ${ }^{4,5}$.Sternick cols demonstraram recentemente a possibilidade da ocorrência de morte súbita em chagásicos com eletrocardiograma normal e fração de ejeção normal ao ecocardiograma ${ }^{6}$.

No grupo que apresentou anormalidades contráteis segmentares, observou-se sinais de comprometimento ventricular global, como foi demonstrado pela maior dimensão sistólica ventricular e menor fração de ejeção quando comparados aos pacientes com contratilidade normal. Viotti cols, em um estudo envolvendo 849 pacientes com cardiopatia chagásica crônica, e acompanhados por cerca de 9,9 anos, demonstraram que piora clínica, dimensão sistólica ventricular esquerda e fração de ejeção foram os únicos preditores de mortalidade 7 . Pacientes classificados na FIDC, mas apresentando alteração contrátil segmentar ao ecocardigrama, apresentam sinais de diminuição global da contratilidade ventricular esquerda. Além disso, independente da classificação clínica, pacientes com alteração contrátil ao ecocardiograma demonstram sinais de depressão maior da contratilidade miocárdica, quando comparados com pacientes que apresentam apenas distúrbios isolados da condução elétrica ao $\mathrm{ECG}^{8}$.

Pazin-Filho cols avaliaram o valor prognóstico de anormalidades contráteis segmentares em 59 pacientes com diferentes formas clínicas da doença de Chagas, demonstrando a ocorrência de deteriorização da função cardíaca global em 71,4\% dos pacientes com dissinergia segmentar em comparação com $22,2 \%(p<0,005)$ naqueles pacientes com função contrátil segmentar normal ${ }^{9}$. Rassi cols demonstraram, após análise multivariada, a relevância prognóstica dos achados de anormalidade contrátil segmentar ou global pela ecocardiografia, como preditores de risco de morte por causas cardiovasculares e morte súbita (hazard ratio de 2,46; $\mathrm{p}=0,008$ ). Embora os autores não tenham feito menção da FIDC, os dados refletem a importância prognóstica da identificação de anormalidade contrátil segmentar em pacientes chagásicos ${ }^{10}$.

O Doppler tecidual demonstrou um comprometimento da contratilidade longitudinal em quase todas as paredes avaliadas, mesmo quando o ecocardiograma mostrasse uma predominância de envolvimento apical, denotando um maior dano miocárdico neste grupo de pacientes. Estudos prévios publicados pelo nosso grupo têm demonstrado a superioridade desta técnica no diagnóstico de anormalidades funcionais precoces na doença de Chagas ${ }^{11-13}$.

A arritmia ventricular representa um aspecto predominante na doença de Chagas. A existência de arritmia ventricular na FIDC tem sido extensivamente demonstrada, sugerindo-se que sua presença esteja relacionada a pior prognóstico ${ }^{2,5,6}$. O presente estudo demonstrou a existência de associação entre anormalidade contrátil e arritmia ventricular na FIDC, indicando que estes pacientes possam representar subgrupo com maior envolvimento cardíaco, e sendo a presença de anormalidade contrátil segmentar um substrato anatômico para o surgimento de um foco arrítmico, mesmo em um coração com função global preservada.

Nossos dados estão em concordância com os relatados por Pedrosa cols, que estudaram 71 pacientes divididos em quatro grupos de acordo com o comprometimento miocárdico, sendo que o grupo IA representava pacientes com ECG e ecocardiograma normais; o grupo IB continha pacientes com ECG normal e alterações contráteis ao ecocardiograma; o grupo II com ECG e Eco anormais e grupo III com ECG e ECO anormais e sinais de insuficiência cardíaca. O Holter demonstrou que cerca de $56 \%$ dos pacientes do grupo IA não apresentaram arritmias ventriculares. No grupo IB, 37,5\% não apresentaram arritmias ventriculares e $25 \%$ apresentaram arritmias ventriculares polimórficas frequentes ao Holter. Em relação a pares de extrassístoles ventriculares (EV), também se observou aumento de sua incidência com a piora do grau de lesão miocárdica. Nenhum paciente do grupo IA apresentou pares de EV ao Holter, sendo que a incidência dessa arritmia no grupo IB foi de $12,5 \%$, demonstrando o aumento da incidência de 
arritmia ventricular quando se avança o grau de comprometimento miocárdico. Presença de taquicardia ventricular não sustentada foi encontrada apenas nos grupos II e III ${ }^{14}$.

Concluindo, pacientes chagásicos na forma indeterminada, que apresentam distúrbio contrátil segmentar à ecocardiografia, podem apresentar frequência maior de arritmia ventricular ao Holter, denotando sinais de um maior comprometimento miocárdico e elétrico do coração. A despeito da heterogeneidade do desempenho cardíaco de pacientes chagásicos com a forma indeterminada, quando avaliados por métodos propedêuticos mais sensíveis, o conceito desta forma continua válido e útil pelo menos em termos coletivos e epidemiológicos. Contudo, pacientes na forma indeterminada podem apresentar alterações morfológicas e funcionais com potencial significado prognóstico, sugerindo que apresentem comportamento diferenciado em relação àqueles pacientes que não apresentaram qualquer alteração morfofuncional cardíaca, mesmo quando submetidos à extensa avaliação. O significado prognóstico e as implicações terapêuticas da existência dessas alterações morfológicas e funcionais, na forma indeterminada da doença de Chagas, devem ser melhor avaliados em estudos prospectivos.

\section{CONFLITO DE INTERESSE}

Os autores declaram não haver nenhum tipo de conflito de interesse no desenvolvimento do estudo.

\section{SUPORTE FINANCEIRO}

Conselho Nacional de Desenvolvimento Científico e Tecnológico (CNPq).

\section{REFERÊNCIAS}

1. Schmunis GA. American Trypanosomiasis as a public health problem In: Pan American Health Organization, editor. Chagas' disease and the nervous system. Washington (DC): Pan American Health Organization; 1994. p. 3-29.

2. Ribeiro ALP, Rocha MOC. Forma indeterminada da doença de Chagas: considerações acerca do diagnóstico e do prognóstico. Rev Soc Bras Med Trop 1998; 31:301-314.

3. Ortiz J, Barreto AC, Matsumoto AY, Monaco CA, Ianni B, Marotta RH, et al. Alteração contrátil segmentar na forma indeterminada da doença de Chagas. Estudo ecocardiográfico. Arq Bras Cardiol 1987; 49:217-220.

4. Bestetti RB, Freitas OC, Muccillo G, Oliveira JS. Clinical and morphological characteristics associated with sudden cardiac death in patients with Chagas' disease. Eur Heart J 1993; 14:1610-1614.

5. Barreto AC, Belloti G, Sosa E, Grupi C, Mady C, Ianni BM, et al. Arritmias e a forma indeterminada da doença de Chagas. Arq Bras Cardiol 1986; 47:197-199.

6. Sternick EB, Martinelli M, Sampaio RC, Gerken LM, Teixeira RA, Scarpelli R, et al. Sudden Cardiac Death in Patients with Chagas Heart Disease and Preserved Left Ventricular Function. J Cardiovasc Electrophysiol 2006; 17:113-116.

7. Viotti RJ, Vigliano C, Laucella S, Lococo B, Petti M, Bertocchi G, et al. Value of echocardiography for diagnosis and prognosis of chronic Chagas disease cardiomyopathy without heart failure. Heart 2004; 90:655-660.

8. Almeida-Filho OC, Maciel BC, Schmidt A, Pazin-Filho A, Marin-Neto JA. Minor segmental dyssynergy reflects extensive myocardial damage and global left ventricle dysfunction in chronic Chagas disease. J Am Soc Echocardiogr 2002; 15:610-616.

9. Pazin-Filho A, Romano MM, Almeida-Filho OC, Furuta MS, Viviani LF, Schmidt A, et al. Minor segmental wall motion abnormalities detected in patients with Chagas' disease have adverse prognostic implications. Braz J Med Biol Res 2006; 39:483-487.
10. Rassi Jr A, Rassi A, Little WC, Xavier SC, Rassi SG, Rassi AG, et al. Development and validation of a risk score for predicting death in Chagas' heart disease. N Engl J Med 2006; 355:799-808.

11. Barros MV, Rocha MO, Ribeiro AL, Machado FS. Doppler tissue imaging to evaluate early myocardium damage in patients with undetermined form of Chagas' disease and normal echocardiogram. Echocardiography 2001; 18:131-136.

12. Barros MV, Ribeiro AL, Machado FS, Rocha MO. Doppler tissue imaging to assess systolic function in Chagas' disease. Arq Bras Cardiol 2003; 80:36-35.

13. Barros MV, Machado FS, Ribeiro AL, Costa-Rocha MO. Detection of early right ventricular dysfunction in Chagas disease using Doppler tissue imaging. J Am Soc Echocardiogr 2002; 15:1197-1201.

14. Pedrosa RC, Campos MC. Teste ergométrico e o Holter de 24 horas na detecção de arritmias ventriculares complexas em diferentes estádios da cardiopatia chagásica crônica. Rev Soc Bras Med Trop 2004; 37:376-383. 